\title{
Generalization of New Continuous Functions in Topological Spaces
}

\author{
P. G. PATIL \\ Department of Mathematics, \\ SKSVM Agadi College of \\ Engg. and Techn., \\ Laxmeshwar-582 116, \\ Karnataka State, India. \\ pgpatil01@gmail.com
}

\author{
T. D. RAyAnAgOUdAR \\ Department of Mathematics, \\ Govt.First Grade College, \\ Annigeri-582 116, \\ Karnataka State, India. \\ rgoudar1980@gmail.com
}

\author{
S. S. BenChalli \\ Department of Mathematics, \\ Karnatak University, \\ Dharwad-580 003 \\ Karnataka State, India. \\ benchalliss@gmail.com
}

\begin{abstract}
In this paper, $\omega \alpha$-closed sets and $\omega \alpha$-open sets are used to define and investigate the new classes of functions namely somewhat $\omega \alpha$-continuous functions and totally $\omega \alpha$ continuous functions.
\end{abstract}

\section{RESUMEN}

En este artículo conjuntos cerrados- $\omega \alpha$ y abiertos- $\omega \alpha$ se usan para definir e investigar las clases de nuevas funciones continuas $\omega \alpha$ y totalmente continuas $\omega \alpha$.

Keywords and Phrases: $\omega \alpha$-closed, $\omega \alpha$-open, $\omega \alpha$-continuous, somewhat $\omega \alpha$-continuous and totally $\omega \alpha$ - continuous functions.

2010 AMS Mathematics Subject Classification: 54C08, 54C10. 


\section{Introduction}

Recent progress in study of charactreization and generalization of continuity has been done by means of several generalized closed sets. As a generalization of closed sets $\omega \alpha$-closed sets were introduced and studied by Benchalli.et.al[1].

The concepts of feebly continuous functions and feebly open functions were introduced by Zdenek Frolik[2]. Gentry and Hoyle[3] introduced and studied the concepts of somewhat continuous functions and somewhat open functions. Recently, Santhileela and Balasubramanian[8] introduced and studied the concepts of somewhat semi continuous functions and somewhat semi open functions. In this paper, we will continue the study of related functions with $\omega \alpha$-closed and $\omega \alpha$-open sets. We introduce and characterize the concept of somewhat $\omega \alpha$-contnuous and totally $\omega \alpha$-continuous functions.

\section{Preliminaries}

Throughout this paper $(X, \tau),(Y, \sigma)$ and $(Z, \eta)$ (or simply $X, Y$ and $Z$ ) represent topological spaces on which no separation axioms are assumed unless otherwise mentioned.For a subset $A$ of $(X, \tau)$, $\operatorname{cl}(A), \operatorname{int}(A), \alpha \operatorname{cl}(A)$ and $A^{c}$ denote the closure of $A$, inerior of $A$, the $\alpha$-closure of $A$ and the compliment of $A$ in $X$ respecively.

We recall the following definitions, which are usefull in the sequel.Before entering into our work we recall the following definitions from various authors.

Definition 2.1. A subset $A$ of a topological space $(X, \tau)$ is called semi-open [5] (resp. $\alpha$-open/6]) if $A \subseteq \operatorname{cl}(\operatorname{Int}(A))$ (resp $A \subseteq \operatorname{Int}(\operatorname{cl}(\operatorname{Int}(A)))$. The compliment of semi-open (resp. $\alpha$-open) is called semi-closed(resp. $\alpha$-closed).

Definition 2.2. A subset $A$ of a topological space $(\mathrm{X}, \tau)$ is called $\omega \alpha$-closed $[1]$ if $\alpha \mathrm{cl}(\mathrm{A}) \subseteq \mathrm{U}$ whenever $\mathrm{A} \subset \mathrm{U}$ and $\mathrm{U}$ is $\omega$-open in $\mathrm{X}$. The compliment of $\omega \alpha$-closed set is $\omega \alpha$-open.

The family of all $\omega \alpha$-closed sets of $X$ is denoted by $\tau_{\omega \alpha}^{*}$. In [7], we showed that $\tau_{\omega \alpha}^{*}$ forms a topology on $X$.

Definition 2.3. A function $\mathrm{f}:(\mathrm{X}, \tau) \rightarrow(\mathrm{Y}, \sigma)$ is is said to be $\omega \alpha$-continuous [7] if the inverse image of every open set in $\mathrm{Y}$ is $\omega \alpha$-open in $\mathrm{X}$.

Definition 2.4. A function $\mathrm{f}:(\mathrm{X}, \tau) \rightarrow(\mathrm{Y}, \sigma)$ is is said to be perfectly $\omega \alpha$-continuous [7] if the inverse image of every $\omega \alpha$ open set in $\mathrm{Y}$ is clopen in $\mathrm{X}$.

Definition 2.5. A function $\mathrm{f}:(\mathrm{X}, \tau) \rightarrow(\mathrm{Y}, \sigma)$ is is said to be somewhat-continuous [3](resp.somewhat semi-continuous[8]) if for $\mathrm{U} \in \sigma$ and $\mathrm{f}^{-1}(\mathrm{U}) \neq \phi$ there exists an open (resp.semi open) set $\mathrm{V}$ in $\mathrm{X}$ such that $\mathrm{V} \neq \phi$ and $\mathrm{V} \subseteq \mathrm{f}^{-1}(\mathrm{U})$. 
Remark 2.6. Every somewhat continuous function is somewhat semi continuous but converse need not true in general[8].

Definition 2.7. A function $\mathrm{f}:(\mathrm{X}, \tau) \rightarrow(\mathrm{Y}, \sigma)$ is said to be somewhat-open [3](resp.somewhat semi-open[8]) function provided that for $\mathrm{U} \in \tau$ and $\mathrm{U} \neq \phi$, there exists an open (resp.semi open) set $\mathrm{V}$ in $\mathrm{Y}$ such that $\mathrm{V} \neq \phi$ and $\mathrm{V} \subseteq \mathrm{f}^{-1}(\mathrm{U})$.

Remark 2.8. Every somewhat open function is somewhat semi open function but the converse need not be true in general[8/.

\section{Somewhat $\omega \alpha$ - Continuous functions}

In this section, we introduce a new class of functions called somewhat $\omega \alpha$-continuous functions using $\omega \alpha$-closed sets and obtain some of their characterizations.

Definition 3.1. A function $\mathrm{f}:(\mathrm{X}, \tau) \rightarrow(\mathrm{Y}, \sigma)$ is said to be Somewhat $\omega \alpha$ - continuous if for every open set $\mathrm{U}$ in $\mathrm{Y}$ and $\mathrm{f}^{-1}(\mathrm{U}) \neq \phi$, there exists $\omega \alpha$-open set $\mathrm{V}$ in $\mathrm{X}$ such that $\mathrm{V} \neq \phi$ and $\mathrm{V} \subseteq \mathrm{f}^{-1}(\mathrm{U})$

Example 3.2. Let $X=Y=\{p, q\}, \tau=\{X, \phi$,$\} and \sigma=\{X, \phi,\{p\}\}$. The identity function $\mathrm{f}:(\mathrm{X}, \tau) \rightarrow(\mathrm{Y}, \sigma)$ is somewhat $\omega \alpha$-continuous function.

Theorem 3.3. Every somewhat continuous function is somewhat $\omega \alpha$-continuous but converse need not true in general.

Example 3.4. In Example 3.2, $f$ is somewhat $\omega \alpha$-continuous but not somewhat continuous.

Remark 3.5. The concept of somewhat $\omega \alpha$-continuous and somewhat semi-continuous functions are independet as seen from the following examples.

Example 3.6. In Example 3.2, is somewhat $\omega \alpha$-continuous but not somewhat-semi continuous.

Example 3.7. Let $\mathrm{X}=\mathrm{Y}=\{\mathrm{a}, \mathrm{b}, \mathrm{c}\}, \tau=\{\mathrm{X}, \phi,\{\mathrm{a}, \mathrm{b}\}\}$ and $\sigma=\{\mathrm{X}, \phi,\{\mathrm{a}\}\}$. Then the identity map $\mathrm{f}:(\mathrm{X}, \tau) \rightarrow(\mathrm{Y}, \sigma)$ is somewhat-semi continuous but not somewhat $\omega \alpha$-continuous.

Theorem 3.8. If $f:(X, \tau) \rightarrow(Y, \sigma)$ is somewhat $\omega \alpha$-continuous and $g:(Y, \sigma) \rightarrow(Z, \eta)$ is continuous function,then their composition gof is somewhat $\omega \alpha$-continuous function.

Proof. Let $\mathrm{U}$ be an open set in Z.Suppose that $\mathrm{f}^{-1}(\mathrm{U}) \neq \phi$. Since $\mathrm{U}$ is open and $\mathrm{g}$ is continuous, $\mathrm{g}^{-1}(\mathrm{U}) \in \eta$. Suppose that $\mathrm{f}^{-1}\left(\mathrm{~g}^{-1}(\mathrm{U})\right) \neq \phi$. By hypothesis, there exists a $\omega \alpha$-open set $\mathrm{V}$ in $\mathrm{Y}$ such that $V \neq \phi$ and $V \subseteq f^{-1}\left(g^{-1}(U)\right)=(g \circ f)^{-1}(V)$. Therefore gof is somewhat $\omega \alpha$-continuous function.

Remark 3.9. In the above Theorem 3.8, if $\mathrm{f}$ is continuous and $\mathrm{g}$ is somewhat $\omega \alpha$-continuous then their composition gof need not be somewhat $\omega \alpha$-continuous function as seen from the following example. 
Example 3.10. Let $X=Y=Z=\{p, q\}, \tau=\{X, \phi,\{p\}\}, \sigma=\{Y, \phi,\{p\}\}$ and $\eta=\{Z, \phi,\{q\}\}$ Define the functions $\mathrm{f}:(\mathrm{X}, \tau) \rightarrow(\mathrm{Y}, \sigma)$ by $\mathrm{f}(\mathrm{p})=\mathrm{f}(\mathrm{q})=\mathrm{q}$ and $\mathrm{g}:(\mathrm{Y}, \sigma) \rightarrow(\mathrm{Z}, \mathrm{\eta})$ by $\mathrm{g}(\mathrm{p})=\mathrm{q}$ and $\mathrm{g}(\mathrm{q})=\mathrm{p}$.Then clearly $\mathrm{f}$ is continuous function and $\mathrm{g}$ is somewhat $\omega \alpha$-continuous function but their comoposition gof $:(X, \tau) \rightarrow(Z, \eta)$ is not somewhat $\omega \alpha$-continuous function.

Definition 3.11. A subset $M$ of a topological space $X$ is said to be $\omega \alpha$-dense in $X$ if there is no proper $\omega \alpha$-closed set $\mathrm{F}$ in $X$ such that $\mathrm{M} \subset \mathrm{F} \subset \mathrm{X}$.

Theorem 3.12. The following statements are equivalent for a function $\mathrm{f}:(\mathrm{X}, \tau) \rightarrow(\mathrm{Y}, \sigma)$ :

(1) $f$ is somewhat $\omega \alpha$-continuous function

(2) If $\mathrm{F}$ is a closed subset of $\mathrm{Y}$ such that $\mathrm{f}^{-1}(\mathrm{~F}) \neq \mathrm{X}$, then there is a proper $\omega \alpha$-closed subset $\mathrm{D}$ of $\mathrm{X}$ such that $\mathrm{f}^{-1}(\mathrm{~F}) \subset \mathrm{D}$.

(3) If $M$ is a $\omega \alpha$-dense subset of $X$, then $f(M)$ is a dense subset of $Y$.

Proof. (1) $\Rightarrow(2)$ : Let $F$ be a closed subset of $Y$ such that $f^{-1}(F) \neq X$.Then $f^{-1}(Y-F)=X-f^{-1}(f) \neq$ $\phi$. Then from (1) there exists $\omega \alpha$-open set $V$ in $X$ such that $V \neq \phi$ and $V \subset f^{-1}(Y-F)=$ $X-f^{-1}(F)$.This implies $f^{-1}(F) \subset X-V$ and $X-V=D$ is a $\omega \alpha$-closed set in $X$.

$(2) \Rightarrow(3)$ : Let $M$ be any $\omega \alpha$-dense set in $X$. Suppose $f(M)$ is not a dense subset of $Y$, then there exists a proper closed set $F$ in $Y$ such that $f(M) \subset F \subset Y$. This implies $f^{-1}(F) \neq X$. Then from (2) there exists a proper $\omega \alpha$-closed set $D$ such that $M \subset f^{-1}(F) \subset D \subset X$. This contradicts the fact that $M$ is a $\omega \alpha$-dense set in $X$.

$(3) \Rightarrow(2)$ : Suppose $(2)$ is not true. Then there exists a closed setF in $Y$ such that $f^{-1}(F) \neq X$. But there is no proper $\omega \alpha$-closed set $D$ in $X$ such that $f^{-1}(F) \subseteq D$. This means that $f^{-1}(F)$ is $\omega \alpha$-dense in $X$. But from hypothesis $f\left(f^{-1}(F)\right)=F$ must be dense in $Y$, which is contradiction to the choice of $F$.

$(2) \Rightarrow(1)$ :Let $U$ be an open set in $Y$ and $f^{-1}(U) \neq \phi$. Then $f^{-1}(Y-U)=X-f^{-1}(U)=\phi$. Then by hypothesis, there exists a proper $\omega \alpha$-closed set $D$ such that $f^{-1}(Y-U) \subset D$. This implies that $X-D \subset f^{-1}(U)$ and $X-D$ is $\omega \alpha$-open and $X-D \neq \phi$.

Theorem 3.13. Let $\mathrm{f}:(\mathrm{X}, \tau) \rightarrow(\mathrm{Y}, \sigma)$ be a function and $\mathrm{X}=\mathrm{A} \cup \mathrm{B}, \mathrm{A}$ and $\mathrm{B}$ are open subsets of $\mathrm{X}$ such that $(\mathrm{f} / \mathrm{A})$ and $(\mathrm{f} / \mathrm{B})$ are somewhat $\omega \alpha$-continuous functions then $f$ is somewhat $\omega \alpha-$ continuous function.

Proof. Let $U$ be an open set in $Y$ such $\operatorname{that}^{-1}(U) \neq \phi$. Then $(f / A)^{-1}(U) \neq \phi$ or $(f / B)^{-1}(U) \neq \phi$ or both $(\mathrm{f} / \mathrm{A})^{-1}(\mathrm{U}) \neq \phi$ and $(\mathrm{f} / \mathrm{B})^{-1}(\mathrm{U}) \neq \phi$.

case $(i)$ : Suppose $(f / A)^{-1}(U) \neq \phi$. Since $f / A$ is somewhat $\omega \alpha$-continuous, then there exists $\omega \alpha$ open set $V$ in $A$ such that $V \neq \phi$ and $V \subset(f / A)^{-1}(U) \subset f^{-1}(U)$. Since $V$ is $\omega \alpha$-open in $A$ and $A$ is open in $X, V$ is $\omega \alpha$-open $X$. Hence $f$ is somewhat $\omega \alpha$-continuous function.

case $(i i)$ : Suppose $(f / B)^{-1}(U) \neq \phi$. Since $f / B$ is somewhat $\omega \alpha$-continuous, then there exists $\omega \alpha$ open set $V$ in $B$ such that $V \neq \phi$ and $V \subset(f / B)^{-1}(U) \subset f^{-1}(U)$. Since $V$ is $\omega \alpha$-open in $B$ and $B$ 
is open in $X, V$ is $\omega \alpha$-open $X$. Hence $f$ is somewhat $\omega \alpha$-continuous function. case $(i i i)$ : Suppose $(f / A)^{-1}(U) \neq \phi$ and $(f / B)^{-1}(U) \neq \phi$. Follows from case(i) and case(ii).

Theorem 3.14. If $A$ be any set in $X$ and $f:(X, \tau) \rightarrow(Y, \sigma)$ be somewhat $\omega \alpha$-continuous such that $\mathrm{f}(\mathrm{A})$ is dense in $\mathrm{Y}$. Then any extension $\mathrm{F}$ of $f$ is somewhat $\omega \alpha$-continuous.

Proof. Let $U$ be an open set in $Y$ such that $F^{-1}(U) \neq \phi$. Since $f(A) \subset Y$ is dense in $Y$ and $\mathrm{U} \cap \mathrm{f}(\mathrm{A}) \neq \phi$. It follows that $\mathrm{F}^{-1}(\mathrm{U}) \cap A \neq \phi$. That is $\mathrm{f}^{-1}(\mathrm{U}) \cap A \neq \phi$.Hence by hypothesis there exists a $\omega \alpha$-open set $V$ in $A$ such that $V \neq \phi$ and $V \subset f^{-1}(U) \subset F^{-1}(U)$. This implies $F$ is somewhat $\omega \alpha$-continuous.

Definition 3.15. A topological space $X$ is said to be $\omega \alpha$-separable if there exists a countable subset $\mathrm{B}$ of $\mathrm{X}$ which is $\omega \alpha$-dense in $\mathrm{X}$.

Theorem 3.16. Let $\mathrm{f}:(\mathrm{X}, \tau) \rightarrow(\mathrm{Y}, \sigma)$ is somewhat $\omega \alpha$-continuous function.If $\mathrm{X}$ is $\omega \alpha$-separable then $\mathrm{Y}$ is separable.

Proof. Let B be countable subset of $X$ which is $\omega \alpha$-dense in $X$. Then from Theorem $3.12, f(B)$ is dense in $Y$. Since B is countable $f(B)$ is also countable which is dense in $Y$. This implies that $Y$ is separable.

\section{Somewhat $\omega \alpha$-Open Functions}

In this section, we introduce the concept of somewhat $\omega \alpha$-open functions and study some of their characterizations.

Definition 4.1. A function $\mathrm{f}:(\mathrm{X}, \tau) \rightarrow(\mathrm{Y}, \sigma)$ is somewhat $\omega \alpha$-open provided that for open set $\mathrm{U}$ in $\mathrm{X}$ and $\mathrm{U} \neq \phi$ there exists a $\omega \alpha$-open set $\mathrm{V}$ in $\mathrm{Y}$ such that $\mathrm{V} \neq \phi$ and $\mathrm{V} \subseteq \mathrm{f}(\mathrm{U})$.

Example 4.2. Let $\mathrm{X}=\mathrm{Y}=\{\mathrm{a}, \mathrm{b}, \mathrm{c}\}$ and $\tau=\{\mathrm{X}, \phi,\{\mathrm{a}\},\{\mathrm{b}, \mathrm{c}\}\}$ and $\sigma=\{\mathrm{X}, \phi,\{\mathrm{a}\}$. Define a function $\mathrm{f}:(\mathrm{X}, \tau) \rightarrow(\mathrm{Y}, \sigma)$ by $\mathrm{f}(\mathrm{a})=\mathrm{c}, \mathrm{f}(\mathrm{b})=\mathrm{a}$ and $\mathrm{f}(\mathrm{c})=\mathrm{b}$. Then clearly $f$ is somewhat $\omega \alpha$-open.

Theorem 4.3. Every somewhat open function is somewhat $\omega \alpha$-open function but converse need not be true in general.

Example 4.4. In Example 4.2, $f$ is somewhat $\omega \alpha$-open function but not somewhat -open function.

Remark 4.5. Somewhat $\omega \alpha$-open and somewhat semi-open functions are independent of each other as seen from the following examples.

Example 4.6. In Example 4.2, $f$ is somewhat $\omega \alpha$-open function but not somewhat semi-open function. 
Example 4.7. Let $\mathrm{X}=\mathrm{Y}=\{\mathrm{a}, \mathrm{b}, \mathrm{c}\}, \tau=\{\mathrm{X}, \phi,\{\mathrm{b}\},\{\mathrm{a}, \mathrm{c}\}\}$ and $\sigma=\{\mathrm{Y}, \phi,\{\mathrm{a}\},\{\mathrm{b}\},\{\mathrm{a}, \mathrm{b}\}\}$. Then the identity function $\mathrm{f}:(\mathrm{X}, \tau) \rightarrow(\mathrm{Y}, \sigma)$ is somewhat semi-open but not somewhat $\omega \alpha$-open function.

Theorem 4.8. If $\mathrm{f}:(\mathrm{X}, \tau) \rightarrow(\mathrm{Y}, \sigma)$ is open function and $\mathrm{g}:(\mathrm{Y}, \sigma) \rightarrow(\mathrm{Z} . \eta)$ is somewhat $\omega \alpha$-open function,then their composition gof is somewhat $\omega \alpha$-open function.

We have the following characterization.

Theorem 4.9. The following statements are eqivalent for bijective function $\mathrm{f}:(\mathrm{X}, \tau) \rightarrow(\mathrm{Y}, \sigma)$

(1) $f$ is somewhat $\omega \alpha$-open function

(2) If $\mathrm{F}$ is closed subset of $\mathrm{X}$ such that $\mathrm{f}(\mathrm{F}) \neq \mathrm{Y}$, then there exists a $\omega \alpha$-closed subset $\mathrm{D}$ of $\mathrm{Y}$ such that $\mathrm{D} \neq \mathrm{Y}$ and $\mathrm{f}(\mathrm{F}) \subset \mathrm{D}$.

Proof. (1) $\Rightarrow(2)$ :Let $F$ be a closed subset of $X$ such that $f(F) \neq Y$. From (1), there exists a $\omega \alpha$ -open set $V \neq \phi$ in $Y$ such that $V \subset f(X-F)$. Put $D=Y-V$. Clearly $D$ is a $\omega \alpha$-closed in $Y$ and we claim that $D \neq Y$. If $D=Y$, then $V=\phi$ which is a contradiction. Since $V \subset f(X-F)$, $D=Y-V \subset Y-[f(X-F)]=f(F)$.

$(2) \Rightarrow(1)$ :Let $U$ be any non-empty open set in $X$. Put $F=X-U$. Then $F$ is a closed subset of $X$ and $f(X-U)=f(F)=Y-f(U)$ which implies $f(F) \neq \phi$. Therefore by (2) there is a $\omega \alpha$-closed subset $D$ of $Y$ such that $D \neq Y$ and $f(F) \subset D$. Put $V=X-D$, clearly $V$ is $\omega \alpha$-open set and $V \neq \phi$.Further, $V=X-D \subset Y-f(F)=Y-[Y-f(U)]=f(U)$.

Theorem 4.10. If $f:(X, \tau) \rightarrow(Y, \sigma)$ is somewhat $\omega \alpha$-open function and $A$ be any open subset of $X$. Thenf $/ A:(A, \tau / A) \rightarrow(Y, \sigma)$ is also somewhat $\omega \alpha$-open function.

Theorem 4.11. If $\mathrm{f}:(\mathrm{X}, \tau) \rightarrow(\mathrm{Y}, \sigma)$ be a function such that $\mathrm{f} / \mathrm{A}$ and $\mathrm{f} / \mathrm{B}$ are somewhat $\omega \alpha$-open, then $\mathrm{f}$ is somewhat $\omega \alpha$-open function, where $\mathrm{X}=\mathrm{A} \cup \mathrm{B}, \mathrm{A}$ and $\mathrm{B}$ are open subsets of $\mathrm{X}$.

\section{Totally $\omega \alpha$ - Continuous Functions}

In this section, we introduce a new class of functions called totally $\omega \alpha$ - continuous functions and study some of their properties.

Definition 5.1. A function $\mathrm{f}:(\mathrm{X}, \tau) \rightarrow(\mathrm{Y}, \sigma)$ is said to be totally $\omega \alpha$-continuous, if the inverse image of every open subset of $\mathrm{Y}$ is an $\omega \alpha$-clopen subset of $\mathrm{X}$.

Example 5.2. Let $X=Y=\{a, b, c\}, \tau=\{X, \phi,\{a\}\}$ and $\sigma=\{\mathrm{Y}, \phi,\{\mathrm{a}\},\{\mathrm{b}, \mathrm{c}\}\}$. Define a function $\mathrm{f}:(\mathrm{X}, \tau) \rightarrow(\mathrm{Y}, \sigma)$ byf $(\mathrm{a})=\mathrm{b}, \mathrm{f}(\mathrm{b})=\mathrm{a}$ and $\mathrm{f}(\mathrm{c})=\mathrm{c}$. Then $\mathrm{f}$ is totally $\omega \alpha$ - continuous function

Theorem 5.3. Every perfectly $\omega \alpha$ - continuous map is totally $\omega \alpha$ - continuous but converse need not be true in general. 
Proof. Let $f:(X, \tau) \rightarrow(Y, \sigma)$ be a perfectly $\omega \alpha$ - continuous. Let $U$ be an open set in $Y$. Then $U$ is $\omega \alpha$-open in $Y$. Since $f$ is a perfectly $\omega \alpha$ - continuous, $f^{-1}(U)$ is clopen in $X$, implies that $f^{-1}(U)$ is $\omega \alpha$-clopen in $X$.

Example 5.4. In Example 5.2, $\mathrm{f}$ is totally $\omega \alpha$ - continuous but not perfectly $\omega \alpha$-continuous.

Theorem 5.5. Every totally $\omega \alpha$ - continuous function is $\omega \alpha$-continuous but converse need not be true in general.

Example 5.6. Let $X=Y=\{a, b, c\}, \tau=\{X, \phi,\{a\}\}$ and $\sigma=\{\mathrm{Y}, \phi,\{\mathrm{a}\},\{\mathrm{a}, \mathrm{c}\}\}$. Then the identity function $\mathrm{f}:(\mathrm{X}, \tau) \rightarrow(\mathrm{Y}, \sigma)$ is $\omega \alpha$-continuous function but not totally $\omega \alpha$ - continuous function.

Remark 5.7. It is clear that the totally $\omega \alpha$ - continuous function is stronger than $\omega \alpha$ - continuous and weaker than perfectly $\omega \alpha$ - continuous.

Theorem 5.8. If $\mathrm{f}:(\mathrm{X}, \tau) \rightarrow(\mathrm{Y}, \sigma)$ is totally $\omega \alpha$ - continuous function from an $\omega \alpha$-connected space $\mathrm{X}$ in to $\mathrm{Y}$, then $\mathrm{Y}$ is an indiscrete space.

Proof. Suppose that $Y$ is not indiscrete space. Let $A$ be a proper non-empty open subset of $Y$. Then $f^{-1}(A)$ is a non-empty proper $\omega \alpha$ - clopen subset of $X$ which is contradiction to the fact that $\mathrm{X}$ is $\omega \alpha$-connected.

Definition 5.9. A topological space $\mathrm{X}$ is said to be $\omega \alpha_{2}$-space [7], if for every pair of distinct points $\mathrm{x}$ and $\mathrm{y}$ in $\mathrm{X}$, there exists $\omega \alpha$-open sets $\mathrm{M}$ and $\mathrm{N}$ such that $\mathrm{x} \in \mathrm{N}, \mathrm{y} \in \mathrm{M}$ and $\mathrm{M} \cap \mathrm{N}=\phi$.

Theorem 5.10. Let $\mathrm{f}:(\mathrm{X}, \tau) \rightarrow(\mathrm{Y}, \sigma)$ be totally $\omega \alpha$ - continuous injection map. If $Y$ is $\mathrm{T}_{0}$, then $\mathrm{X}$ is $\omega \alpha_{2}$-space.

Proof. Let $x$ and $y$ be any pair of distinct points of $X$. Then $f(x) \neq f(y)$. Then there exists an open set $U$ containing $f(x)$ but notf $(y)$. Since $Y$ is $T_{0}$. Then $x \notin f^{-1}(U)$ and $y \notin f^{-1}(U)$. Since $f$ is totally $\omega \alpha$ - continuous, $f^{-1}(U)$ is an $\omega \alpha$-clopen subset of $X$. Also $x \in f^{-1}(U)$ and $y \in\left(f^{-1}(U)\right)^{c}$. Hence $X$ is $\omega \alpha_{2}$-space.

Theorem 5.11. A topological space $\mathrm{X}$ is $\omega \alpha$-connected if and only if every totally $\omega \alpha$-continuous function from a space $\mathrm{X}$ in to any $\mathrm{T}_{0}$-space $\mathrm{Y}$ is a constant function.

Theorem 5.12. Let $\mathrm{f}:(\mathrm{X}, \tau) \rightarrow(\mathrm{Y}, \sigma)$ is totally $\omega \alpha$ - continuous and $\mathrm{Y}$ be $a \mathrm{~T}_{1}$-space. If $\mathrm{A}$ is an $\omega \alpha$-connected subset of $\mathrm{X}$, then $\mathrm{f}(\mathrm{A})$ is a single point.

Theorem 5.13. A function $\mathrm{f}:(\mathrm{X}, \tau) \rightarrow(\mathrm{Y}, \sigma)$ is totally $\omega \alpha$ - continuous at a point $\mathrm{x} \in \mathrm{X}$ if for each open subset $\mathrm{V}$ in $\mathrm{Y}$ containing $\mathrm{f}(\mathrm{X})$, there exists a $\omega \alpha$-clopen subset $\mathrm{U}$ in $\mathrm{X}$ containing $\mathrm{x}$ such that $\mathrm{f}(\mathrm{U}) \subset \mathrm{V}$. 
Proof. Let $\mathrm{V}$ be an open subset of $Y$ and let $x \in \mathrm{f}^{-1}(\mathrm{~V})$. Since $\mathrm{f}(\mathrm{x}) \in \mathrm{V}$, there exists a $\omega \alpha$-clopen

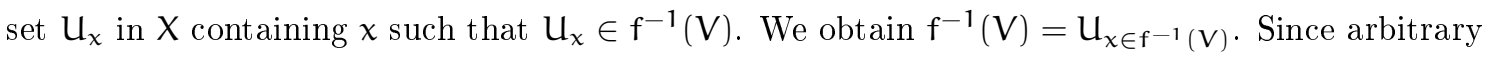
union of $\omega \alpha$-open sets is $\omega \alpha$-open, $f^{-1}(V)$ is $\omega \alpha$-clopen in $X$.

Definition 5.14. Let $\mathrm{X}$ be a topological space. Then the set of all points $\mathrm{y}$ in $\mathrm{X}$ such that $\mathrm{x}$ and $\mathrm{y}$ cannot be separated by a $\omega \alpha$-separation of $\mathrm{X}$ is said to be the quasi $\omega \alpha$-component of $\mathrm{X}$.

Theorem 5.15. Let $\mathrm{f}:(\mathrm{X}, \tau) \rightarrow(\mathrm{Y}, \sigma)$ is totally $\omega \alpha$ - continuous map from a topological space $\mathrm{X}$ in to a $\mathrm{T}_{1}$-space $\mathrm{Y}$, then $\mathrm{f}$ is constant on each quasi $\omega \alpha$-component of $\mathrm{X}$.

Proof. Let $x$ and $y$ be two points of $X$ that lie in the some quasi $\omega \alpha$-component of $X$. Assume that $f(x)=\alpha \neq \beta=f(y)$. Since $Y$ is $T_{1}, \alpha$ is closed in $Y$ and so $\alpha^{c}$ is an open subset in $Y$. Since $f$ is totally $\omega \alpha$-continuous, $f^{-1}(\alpha)$ and $f^{-1}\left(\alpha^{c}\right)$ are disjoint $\omega \alpha$-clopen subsets of $X$. Further $x \in f^{-1}(\alpha)$ and $y \in f^{-1}(\alpha)^{c}$, which is a contradiction in view of the fact that $y$ must belong to every $\omega \alpha$-clopen set containing $x$.

Received: April 2013. Accepted: September 2013.

\section{References}

[1] S.S.Benchalli, P.G.Patil and T.D.Rayanagoudar, $\omega \alpha$-Closed Sets in Topological Spaces,The Global Jl.of Appl.Math.and Math.Sciences, V.2,1-2,(2009),53-63.

[2] Zdenek Frolik,, Remarks concerning the Invariance of Baire Spaces under Mappings, Czech.Math.Jl., II(86)(1961),389-385.

[3] K.R. Gentry and H.B. Hoyle, Somewhat continuous functions, Czech.Math.Jl., 21, No.1 (86) (1971),5-12.

[4] N. Levine, Generalized closed sets in topology, Rend. Circ. Mat. Palermo, 19 (2) (1970), 89-96

[5] N. Levine, Semi-open sets and Semi-continuity in topological spaces, Amer. Math. Monthly, 70 (1963), 36-41.

[6] O.Njastad, On some classes of nearly open sets, Pacific Jl.Math., 15(1965),961-970.

[7] P.G.Patil,, Some Studies in Topological Spaces, Ph.D.Thesis, Karnatak University Dharwad (2007).

[8] D.Santhileela and G Balasubramanian, Somewhat semi continuous and somewhat semi open functions, Bull.Cal.Math.Soc.,94(1)(2002) 41-48. 